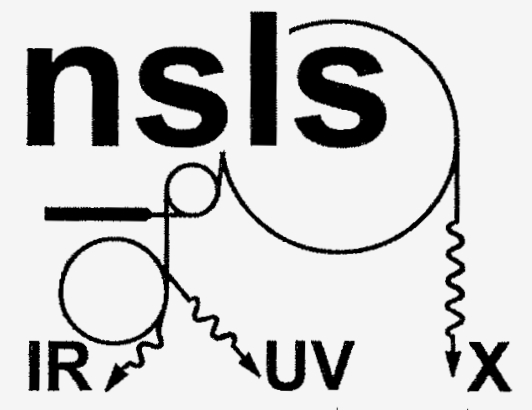

BNL- 65581

Informal Report

\title{
In Vacuum Undulator Task Force Report
}

J. B. Hastings, C.-C. Kao, P. Stefan, S. Burley, G. S. Cargill, M. Chance, S. Dierker, W. Hendrickson, K. Evans-Luderodt and H.-K. Mao

RECEIVED

AUG 171998

OSTI

June 1998

DISTRIBUTION OF THIS DOCUMENT IS UNLIMITED

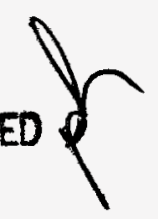

MASTER

\section{NATIONAL SYNCHROTRON LIGHT SOURCE}

\author{
BROOKHAVEN NATIONAL LABORATORY \\ BROOKHAVEN SCIENCE ASSOCIATES \\ Under Cantract No. DE-AC02-98CH10886 with the \\ UNITED STATES DEPARTMENT OF ENERGY
}




\section{DISCLAIMER}

This report was prepared as an account of work sponsored by an agency of the United States Government. Neither the United States Government nor any agency thereof, nor any of their employees, nor any of their contractors, subcontractors, or their employees, makes any warranty, express or implied, or assumes any legal liability or responsibility for the accuracy, completeness, or usefulness of any information, apparatus, product, or process disclosed, or represents that its use would not infringe privately owned rights. Reference herein to any specific commercial product, process, or service by trade name, trademark, manufacturer, or otherwise, does not necessarily constitute or imply its endorsement, recommendation, or favoring by the United States Government or any agency, contractor or subcontractor thereof. The views and opinions of authors expressed herein do not necessarily state or reflect those of the United States Government or any agency, contractor or subcontractor thereof. 


\section{DISCLAIMER}

Portions of this document may be illegible electronic image products. Images are produced from the best available original document. 
BNL- 65581

Informal Report

\section{Vacuum Undulator Task Force Report}

J. B. Hastings, C.-C. Kao, P. Stefan, S. Burley, G. S. Cargill, M. Chance,

S. Dierker, W. Hendrickson, K. Evans-Luderodt, H.-K. Mao

June 1998

National Synchrotron Light Source

Brookhaven National Laboratory, Upton, NY 11973 USA

Work performed under the auspices of the U.S. Department of Energy, under contract DE-AC02-98CH10886 
In Vacuum Undulator Task Force Report

J. B. Hastings, Task Force Chair

National Synchrotron Light Source

Brookhaven National Laboratory

Upton, NY

The Green-Chasman lattice, which is the basis for both NSLS storage rings, was conceived with insertion devices in mind. Long, field-free straight sections were provided in the design. The electron optics were chosen so that these sections had zero dispersion and the effects of new magnetic structures placed in these regions would have minimal effect on the emittance of the electron beam. This design concept has been followed by all highbrightness rings which were built subsequent to the NSLS.

The X-Ray Ring straight sections also have a very small vertical $\beta$ function, in addition to the zero dispersion. This was done to optimize the brightness of wiggler sources. There is a further benefit however. The $\beta$ function determines the beam size and divergence at a particular point in the storage ring lattice. The size is proportional to $\sqrt{\beta}$ and the divergence is proportional to $1 / \sqrt{\beta}$. Thus the electron beam is very small at the center of the X-Ray Ring straight sections. In the initial development of the insertion device program, no specific advantage was taken of this feature. Of the eight straight sections in the X-Ray Ring lattice, five are readily available for magnetic insertion devices and the remaining three are dedicated to radio-frequency drive cavities (2) and injection (1).

Historically the NSLS has been active in R\&D for state-of-the-art electron beams, photon beams and $x$-ray optics. One of the available straight sections has therefore been dedicated to insertion device R\&D. Over the past five to seven years a program aimed at exploiting the very small vertical $\beta$ function in the straight sections has yielded first a prototype small gap undulator (PSGU) ${ }^{1}$ and then an in-vacuum undulator (IVUN) ${ }^{1}$. The basic idea for these devices is to make the magnet gap small so one can make the magnet period small as well, producing hard $\mathrm{x}$-rays from a medium energy electron storage ring like the NSLS X-Ray Ring, without compromising performance. The relationship between magnet period and the photon wavelength of the undulator fundamental is given below:

$$
\lambda=\frac{\lambda u}{2 \gamma^{2}}\left(1+K^{2} / 2\right)
$$

where $\lambda$ is the photon wavelength, $\lambda_{u}$ is the magnet wavelength (period), $\gamma$ is the electron beam energy in units of the electron rest energy, and $K$ is the magnetic strength parameter, which for the devices considered here is of order 1 . Thus, with the NSLS XRay Ring electron beam energy of $2.5 \mathrm{GeV}$ and a magnetic period of $1 \mathrm{~cm}$, a photon wavelength of $3.1 \AA(4.0 \mathrm{keV})$ is produced.

The PSGU study provided independent control of the magnet and vacuum chamber gaps, with the magnet located outside the vacuum envelope of the storage ring. This permitted 
independent evaluation of the effect of a small vacuum gap and small magnet gap on the stored electron beam. The conclusion of these studies was that small gaps can be achieved with minimal effect on storage ring performance. Furthermore, magnetic structures with periods less than $2 \mathrm{~cm}$ can be built to high tolerances and the photon beams they yield are quantitatively in agreement with calculation.

These results encouraged us to pursue a collaboration to build an in-vacuum undulator that would yield first harmonic radiation well into the $x$-ray region of the spectrum. The Spring8 team in Harima, Japan are amongst the world leaders in insertion devices and as partners in the IVUN collaboration they constructed the magnetic arrays for the IVUN project. The $11 \mathrm{~mm}$ period yields a fundamental energy of nearly $4.6 \mathrm{keV}$. As with the PSGU, the project demonstrated that undulator radiation, now from an in-vacuum device and at $x$-ray energies, can be produced at NSLS. The impact of this program of insertion device research at NSLS is significant. Because of the very short periods, the overall length of the entire IVUN assembly is under a meter. This opens up the possibility of placing these devices between the X-Ray Ring RF cavities which produces two additional locations for insertion device sources.

The IVUN sources attain a brightness similar to the existing hybrid wigglers in $\times 21$ and $X 25$. They radiate significantly lower total power than the wigglers but produce higher power densities. They provide undulator rather than wiggler spectra, as can be seen in Fig. 1. Because of the small gaps and small periods there is not much tunability in these devices and they will have to be purpose-built for a specific scientific program. The original IVUN parameters were chosen for in-elastic $x$-ray scattering, similar to the scientific program on $\times 21$. This put the fundamental at $4.6 \mathrm{keV}$ and the third harmonic at $13.8 \mathrm{keV}$.

The question that this new possible insertion device poses is "what science programs can best take advantage of this new insertion device source?". To answer this, a task force was formed by M. Hart, NSLS Department Chair and charged with identifying viable scientific programs that could seek outside funding to construct IVUN beamlines. The membership of the task force was composed of NSLS staff, J. B. Hastings, C.-C. Kao and P. Stefan as well as S. Burley, Rockefeller University, G.S. Cargill, Lehigh University, M. Chance, Einstein College of Medicine, S. Dierker, Univ. of Michigan, W. Hendrickson, Columbia University, K. Evans-Luderodt, Lucent Technology and H.-K. Mao, Carnegie Geophysical Institution.

The task force concentrated on experimental programs that are presently being pursued on new insertion devices worldwide. For example, $x$-ray photon correlation spectroscopy, which takes advantage of the large coherent flux from undulator sources, was considered. However, this program was not considered as the highest priority because, although as an NSLS source the IVUN is amongst the brightest in the hard $x$-ray spectral region, it is still down by several orders of magnitude compared to third-generation machines. In the area of high pressure diamond cell research as well, the need for high energy photons $(>20$ $\mathrm{KeV}$ in general) also puts an IVUN source at a distinct disadvantage.

The general area of protein crystallography, however, is ideal for the IVUN source. The gain in flux-on-sample is significant when compared to NSLS bending magnet sources, in 
particular for small crystals and/or very large unit cells. When the X-Ray Ring running energy is finally settled between 2.5 and $2.8 \mathrm{GeV}$, an IVUN source can be designed to work effectively for MAD studies as well, concentrating for example on the Se $K$ edge.

The unique electron beam optics that makes the IVUN possible in the first place, namely an extremely small vertical $\beta$ function, also makes the IVUN ideal as a source for microdiffraction. The vertical full-width-at-half maximum of the source is approximately 16 microns. Simple $x$-ray optics have the potential of delivering high intensity $x$-ray beams with sub-micron dimensions. This is an exciting possibility for materials characterization in many areas of current interest.

The list of potential scientific programs investigated by the task force is by no means exhaustive. Rather, its findings, which are described in detail in the two appendices, provide two clear scientific disciplines that would benefit significantly from an IVUN source. 


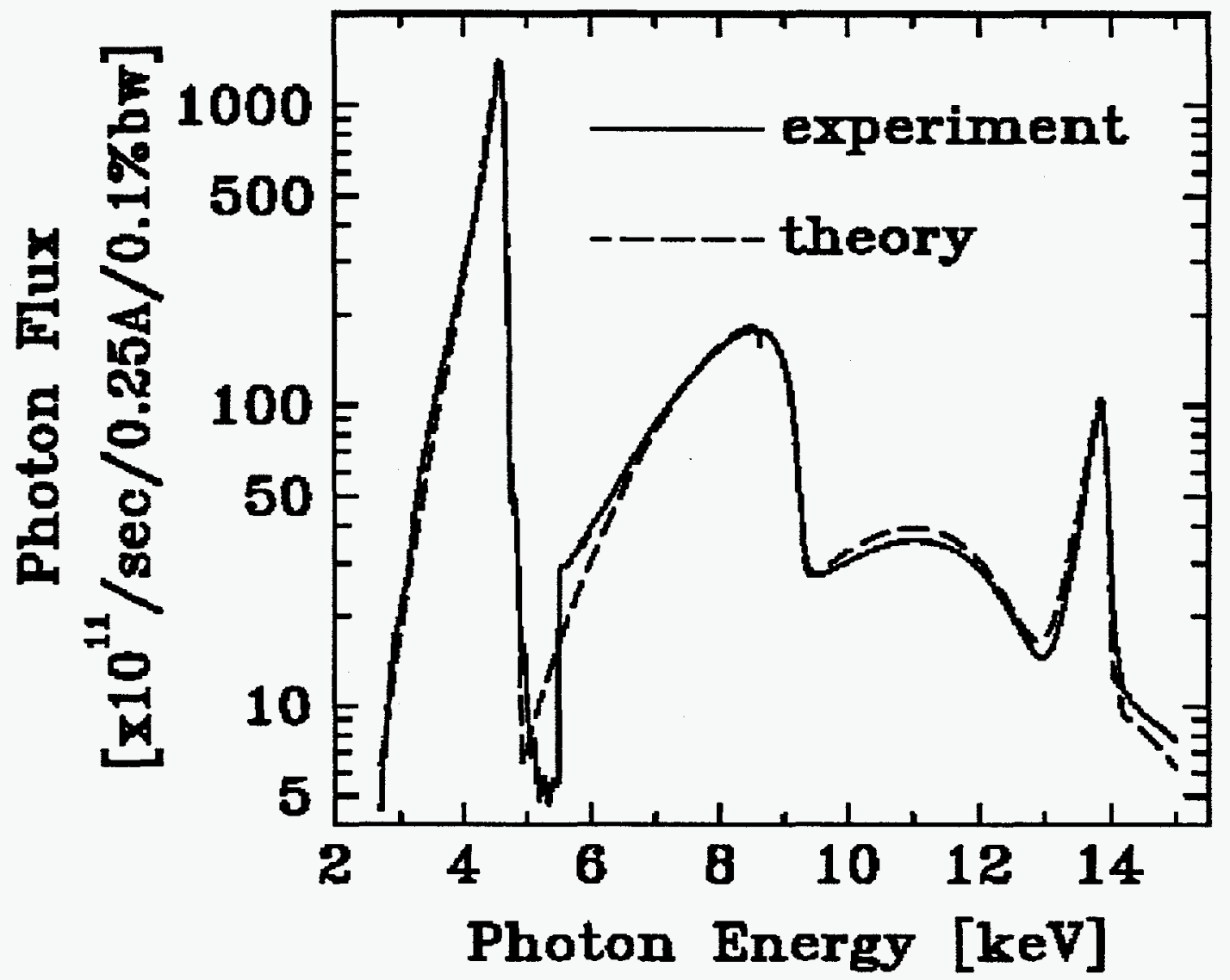

Figure 1. The measured and calculated spectrum from the IVUN in the $\times 13$ straight section. The magnet period is $1.1 \mathrm{~cm}$ and the gap is $0.331 \mathrm{~cm}$. The calculation was done with the URGENT code (R.P. Walker, B. Diviacco, Rev. Sci. Instr. 63(1), $392(1992))$. 
References

1. P.M. Stefan, S. Krinsky, G. Rakowsky, L. Solomon and D. Lynch, "Small Gap Undulator Research at NSLS: Concepts and Results", Nucl. Instr. Meth., in press. BNL65092 
Use of an In-Vacuum Small-Gap Undulator (IVUN) For Microdiffraction

\author{
G. S. Cargill III, Lehigh University \\ K. Evans-Lutterodt, Lucent Technologies \\ J. L. Jordan-Sweet, IBM Research
}

\title{
I. Introduction
}

The three areas for possible use of IVUN considered in materials physics were microdiffraction, speckle studies, and high-pressure studies. Representatives from these areas on the IVUN Task Force were G. S. Cargill III and K. Evans-Lutterodt (microdiffraction); S. Dierker (speckle); and H.-K. Mao (high pressure). Although the properties of the IVUN source would be useful for some speckle and high pressure experiments, groups working in these areas are presently well served by undulator sources at APS (speckle experiments) or wiggler sources at NSLS (high pressure experiments on $X 17 C)$. It seems unlikely that these communities would organize to develop support for IVUN-based beamlines at NSLS for these types of experiments. In contrast, there is considerable interest in possible use of IVUN for microdiffraction, building on work already underway on NSLS bending magnet beamlines ( $\times 26 \mathrm{C}$, Cargill et al.; $\times 16$, Evans-Lutterodt et al., and $\times 20$, Jordan-Sweet et al.). Therefore, the remainder of this report, prepared by G. S. Cargill III, K. Evans-Lutterodt, and J. L. Jordan-Sweet, focuses on the use of an IVUN for microdiffraction.

\section{Importance of Microdiffraction}

$\mathrm{X}$-ray microdiffraction is used for characterizing a material's crystal structure, or variations in that structure due to strain or other defects, with spatial resolution on the micron scale or smaller. Many important current and future applications of microdiffraction are in the integrated circuit (IC) industry. IC devices with smallest critical dimensions of order $0.35 \mathrm{~mm}$ are now being manufactured. Measurements of strain, structure variations and other local materials properties on the micron-scale are vital for understanding failure mechanisms and for insuring long term reliability of these devices. For example, better understanding electromigration in polycrystalline metal interconnect lines of IC's requires better measurements of the strain distributions in these lines and of their time evolution. Microdiffraction from the single-crystal silicon within which the IC's devices are fabricated can provide important information about strains and other structure modifications introduced during device fabrication and affecting device reliability and performance.

Other applications, besides microelectronics, include strain measurements for different components of composites, strain distributions around defects such as crack tips in stressed materials, structure determination for minute crystals embedded in other materials, and microfluorescence mapping of elemental compositions of materials with high sensitivity and high spatial resolution. 
With the present NSLS bending magnet sources and currently available optics, many microdiffraction measurements with $5 \mathrm{~mm}$ to $10 \mathrm{~mm}$ spatial resolution can be carried out. The much greater brightness and much smaller source size for IVUN should allow enable microdiffraction with $0.1 \mathrm{~mm}$ spatial resolution. This will open up possibilities for applying microdiffraction in many other areas.

\section{Electromigration: A Current Microdiffraction Research Problem}

A key part of any integrated circuit is the wiring that connects up the devices. This wiring, referred to as "interconnects," is created by patterning of multi-level thin film metal depositions into narrow strips. In order to increase device density on a chip, the crosssectional area of wires must decrease, leading to an increase in the already substantial current density in these wires. The wires are typically composed of aluminum grains, doped with copper or silicon, with a grain size of order the thickness of the deposited film, which is typically $1 \mathrm{~mm}$. There is some texture to these grains.

They typically grow with a [111] axis almost perpendicular to the surface, but with a distribution half-width of 6 degrees if grown on bare $\mathrm{SiO}_{2}$, and 3 degrees if grown on Ti/TiN.

Electromigration is electric-current-induced atomic diffusion. Due to momentum transfer between the electron current and the atoms in the lattice, there is a biased self-diffusion of the atoms. The momentum transfer is commonly referred to as the "electronic wind", and it leads to an excess of $\mathrm{Al}$ atoms moving to one end of the wire. The effectiveness of this electric-current-induced diffusion of atoms is affected by the grain size and orientation distributions, relative to the wire dimensions. Electromigration causes stress in conductor lines in addition to any residual stress from the manufacturing process, which is usually a result of the differential expansion coefficients of the silicon substrate and the aluminum wires. Thus, in the presence of a steady state electronic current along a wire an anisotropic stress gradient is expected to develop along the wire. If the stress exceeds certain critical values, voids develop in the wire, or cracks occur in the surrounding passivation, either of which can lead to failure of the circuit.

There are two primary reasons that $x$-ray microdiffraction is well suited to study this problem. The first is that it can be used to extract the average interatomic spacing as a function of location. This allows one to deduce the strain in a non-destructive fashion for different locations along a wire. The second reason is that $\mathrm{x}$-rays can study buried objects in-situ. The most technologically relevant electromigration induced failures are those for which the interconnects are enclosed in a rigid $\mathrm{SiO}_{2}$ coating, which serves as an insulator. If the Al interconnects have no passivation coating, many other probes can be used to study the stress. However, it has been suggested that for the very thin lines, the important path for $\mathrm{Al}$ atom diffusion is along the interconnect/casing interface, which implies that the ideal electromigration experiment will be in the buried structure. X-ray diffraction is one of the few techniques that permit study of buried polycrystalline structures with micron-scale spatial resolution. 
In summary, along any given wire, one has a distribution of Al grains with a distribution of sizes and orientations and stresses. In order to help understand the mechanisms for failure in these wires, suggested experiments include mapping the strain distribution along a wire segment, with and without electronic current flowing, to determine the relationships between grain orientation distributions and avoid or slit formation. An instrument that can do this will also be quite a useful general-purpose instrument and will have many other applications beyond electromigration.

\section{IVUN as A Source for Microdiffraction}

Source characteristics important for microdiffraction and microfluorescence are brightness, source size and energy range,. Small source size, which limits needs for high demagnifications, and high brightness, which increases the flux available in very small beams, are particularly desirable in microdiffraction or microfluorescence experiments. A wide energy range enables diffraction measurements with a correspondingly large range of d-spacings. For $x$-ray microdiffraction, a wide energy range enables diffraction measurements with many different (hkl) reflections without having to rotate the sample. As discussed in separate sections below, this can be very important for strain mapping in polycrystalline solids with high spatial resolution, but it is less important for strain mapping in highly textured polycrystais or in single crystals. The high brightness and small source size expected with IVUN are very well suited for microdiffraction. The IVUN energy ranges, as discussed below, have both advantages and disadvantages for these types of experiments.

Source size and brightness. One of the key advantages of the proposed locations of the IVUN devices is that they are points in the ring where the electron beam vertical size is a minimum, about $6 \mathrm{~mm}$. Because of IVUN's short length, its effective source size is smaller than for more traditional, longer insertion devices. With $x$-ray optical elements giving a demagnification factor of 100 , a microbeam of $0.06 \mathrm{~mm}$ height could be produced. This is smaller than the minimum feature size expected in IC's during the next decade, based on the Semiconductor Industry Association's Semiconductor Technology Roadmap. With the high brightness expected from IVUN, enough photons should be available for diffraction or fluorescence measurements using such with deep submicron beam sizes.

The vertical source divergence, about $2 \times 10^{-5}$ rad, will be increased with $\times 100$ demagnification optics to $2 \times 10^{-3}$ rad at the sample. For diffraction from $0.5 \mathrm{~mm}$ grains, finite size broadening is about $0.5 \times 10^{-3} \mathrm{rad}$, to which will be added inhomogeneous strain broadening. Therefore, the incident vertical divergence is reasonably well matched to the sample's angular acceptance for microdiffraction.

The horizontal source size, $400 \mathrm{~mm}$, and source divergence, $260 \times 10^{-6}$ rad, are problematic. With acceptance of the full horizontal beam by $100 \times$ demagnification optics, the horizontal size would be $4 \mathrm{~mm}$ and the horizontal divergence would be $260 x$ $10^{-3}$ rad at the sample. Although for many microdiffraction experiments an asymmetric 
beam of $0.08 \mathrm{~mm} \times 4 \mathrm{~mm}$ would be acceptable, a more symmetrical beam shape and smaller beam divergence could be obtained by reducing the effective horizontal source size and divergence with a pair of white beam slits. We recommend that a pair of slits mounted as close as possible to the source be provided as part of this beamline.

In passing, we note that the source size at ALS is $20 \mathrm{~mm}$ and at APS is $40 \mathrm{~mm}$ at all the insertion devices. At the NSLS, the favorable $6 \mathrm{~mm}$ vertical size is only at the proposed IVUN locations. Since the divergence is larger at these points, the total flux into a spot that a given pair of optics would achieve at the NSLS would be smaller than the same optics at the APS, but the spot size would be smaller.

Heat load. A second key feature of IVUN for microdiffraction is that its output energy distribution, concentrated in fundamental, second harmonic, and third harmonic regions, reduces the heat load on the $x$-ray optics compared with continuous spectrum, wiggler sources of similar brightness. Reduced heat load will ease the job of providing $x$-ray optics with final beam positions of the required stability.

\section{IVUN For Laue, Energy Dispersive and Scanned-Energy Microdiffraction}

Maintaining high spatial resolution in microdiffraction studies precludes rotating the sample to orient it for diffraction from different (hkl) planes in a small single crystal, which may be a single grain of a polycrystalline material, or for diffraction from grains with different orientations within a polycrystalline sample. Rotations are always accompanied by undesired translations because of mechanical imperfections in bearings and because of alignment errors. The alternative to rotating the sample is to rotate the detector and scan the $x$-ray energy, either before or after the sample. For this reason, the range of $x$-ray energies available from IVUN is an important issue for microdiffraction.

Wang, Cargill, Noyan, et al. $(1,2)$ have used white radiation from bending magnet and wiggler beamlines with an energy dispersive detector for these types of measurements. Evans-Lutterodt, Isaacs, Marcus, et al. $(2,3)$ have used bending magnet white radiation incident on a monochromator before the diffracting sample. This monochromator was scanned to obtain different energy $x$-rays. They could use an area detector, since they did not need to use an energy dispersive detector. For measurements on individual grains within polycrystalline materials, Laue diffraction with area detectors will be important for determining orientations of grains before measuring d-spacings for different (hkl) planes of the individual grains, e.g. in grain-by-grain strain and orientation mapping by $x$-ray microdiffraction.

With IVUN, only narrow energy ranges are available near the fundamental, second harmonic, and third harmonic energies, for fixed gap spacing. Moving away from the energies of maximum intensity for the fundamental, second harmonic, and third harmonics, the intensities fall by about $30 \%$ from their peak values for $\pm 250 \mathrm{eV}$ for the fundamental, $\pm 700 \mathrm{eV}$ for the second harmonic, and $\pm 100 \mathrm{eV}$ for the third harmonic. Photon flux and brightness for the second and third harmonics are about $15 \%$ and $7 \%$ those of the fundamental. 
Since the gap of the IVUN can be scanned, the energies of the fundamental and harmonics can be shifted over energy regions of about $4.5-5.5 \mathrm{keV}, 8.5-10 \mathrm{keV}$, and $13.8-16$ $\mathrm{keV}$, for the fundamental and for the second and third harmonics. However the flux and brightness fall off by 10 to 20 times as the gap is increased from its minimum to its maximum value.

By using an area detector and by scanning the gap of the IVUN, Laue-like patterns could be obtained. Taking the available energy ranges to be those given above, the reciprocal space volume accessible by scanning the gap of the IVUN for each of the energy ranges is given by

$$
V_{i}=\frac{4 \pi}{3}\left[\left(\frac{1}{\lambda_{\min , i}}\right)^{3}-\left(\frac{1}{\lambda_{\max , i}}\right)^{3}\right]
$$

where $\lambda_{\min , i}$ and $\lambda_{\max , i}$ are the minimum and maximum wavelengths available with good brightness by tuning the IVUN gap for $\mathbf{i}=$ fundamental, second harmonic, and third harmonic. For the energy ranges given above,

$$
V_{\text {tot }}=V_{1}+V_{2}+V_{3}=5.3 \mathrm{~A}^{-3}
$$

If the energy spectrum extended continuously from $4.5 \mathrm{keV}$ to $16 \mathrm{keV}$, the corresponding reciprocal space volume would be $9.7 \AA^{-1}$. Therefore, with the gap-scanned IVUN spectrum, about half the number of Laue peaks would be seen as with a continuous spectrum extending from $4.5 \mathrm{keV}$ to $16 \mathrm{keV}$, e.g. from a bending magnet or wiggler source.

Even with the need to scan the gap spacing and with the limited energy ranges available, it should be possible to develop instrumentation and methods for high spatial resolution diffraction measurements with this source. The high brightness and small source size, particularly for the energies covered by the fundamental emission, make this an exciting possibility. For the fundamental emission, brightness' exceed those of APS bending magnetic lines $(200 \mathrm{ma})$, by up to an order of magnitude. These brightnesses exceed those of NSLS bending magnetic beamlines (500ma) by two to three orders of magnitude for minimum gap spacings. At maximum gap spacings, the IVUN brightnesses are greater by from two to fifty times than those from NSLS bending magnet beamlines. 


\section{IVUN for Monochromatic Microdiffraction}

In addition to Laue or energy dispersive diffraction with a range of energies provided by scanning the IVUN gap spacing, monochromatic diffraction can be used for a number of microbeam measurements at an IVUN beamline. As noted above, it is a challenge to attempt to find diffraction peaks from a micron-sized region of a random polycrystalline sample using only one photon energy because of the "sphere-of-confusion" inherent in 4circle diffractometers. However, there are other classes of samples, such as highly textured metal films or well-characterized silicon wafers supporting devices or patterned overlayers, which lend themselves to the increased accuracy of d-spacing determination available by knowing the photon energy precisely.

The energy range of $8.5-11 \mathrm{kV}$ provided by the second harmonic of the undulator is ideal for the small d-spacing materials of interest to the microelectronics industry. At a given energy in this range, several reflections from silicon, aluminum, copper, or tungsten meet the Bragg condition for reflection at reasonable angles of two-theta. In fact, the lower end of the energy range can be exploited to increase the reflection angle toward 90 degrees, taking advantage of the low spread in $d$ as a function of theta. For deposited metal films, $y$

vs. $\sin ^{2} y$ plots can be determined for a sampling of grains, or for an individual grain provided there is a feedback mechanism to keep the beam on the same grain for each angle $y$. Much insight can be made in comparing local-scale materials parameters to global averages. Grain orientation maps can be made by setting the detector at a particular angle and scanning the sample. For small features deposited on a silicon wafer, strain fields in the silicon can be mapped by setting two-theta to a silicon reflection and scanning the sample (4). This can also be done for reflections from the material in individual features. In this way a topograph can be plotted and identification of faulty devices, or studies of strain in devices, can be performed.

Another use of monochromatic or pink beam from an IVUN is the determination of the structure of a small grain of material imbedded in a matrix of other grains, for example, a superconducting grain in a materials chemist's crucible. A possible way to do this would be to use a highly accurate single rotation stage at 45 degrees to the incident beam and an area detector at 90 degrees.

\section{HOW TO PROCEED TO USE IVUN FOR MICRODIFFRACTION?}

Before deciding whether to develop an IVUN beamline for microdiffraction at NSLS, exploratory experiments should be performed with the IVUN on beamline $\times 13$. With the microdiffraction setup used by Cargill et al. (1), measurements could be made on aluminum conductor lines with energy dispersive detection. Laue patterns could be collected with an area detector and with Polaroid film for comparisons with those from bending magnet beamlines. If an upstream monochromator can be arranged for $X 13$, Evans-Lutterodt et al. and Jordan-Sweet et al. could evaluate the IVUN source with their respective microdiffraction setups.

If no unexpected difficulties are uncovered in these experiments, plans could be developed for an IVUN beamline for microdiffraction studies, as well as other future applications. The 
beamline would have to have a suitable monochromator and mirror, which could be moved into and out of the line as required. Also, the beamline would require a large hutch which could accommodate roll-in microdiffraction instruments.

\section{References}

1) P.-C. Wang, G. S. Cargill III, I. C. Noyan, E. G. Liniger, C.-K. Hu, and K. Y. Lee, "X-Ray Microdiffraction for VLSI," MRS Symp. Proc. 427, 35 (1996); I. C. Noyan, E. G. Liniger, C.-K. Hu, P.-C. Wang, and G. S. Cargill III, "Local and Global Stress Distributions in BEOL Metallization," MRS Symp. Proc. 428, 565 (1996); P.-C. Wang, G. S. Cargill III, I. C. Noyan, and C.-K. Hu, Electromigration-Induced Stress in Aluminum Conductor Lines Measured by X-Ray Microdiffraction," Appl. Phys. Letters, to be published March 16 , 1998.

2) K. Evans-Lutterodt, E. Isaacs, M. Marcus, et al.

3) J. L. Jordan-Sweet, K. Evans-Lutterodt, G. S. Cargill III, M. A. Marcus, and I. C. Noyan, "Microbeam Diffraction at NSLS," NSLS Newsletter, July 1997, p. 7.

4) I. C. Noyan, J. L. Jordan-Sweet, E. G. Liniger, and S. Kaldor, submitted to Appl. Phys. Letters. 


\section{APPENDIX B}

Structure Determination of Macromolecules and their Assemblies Using a Small-Gap Undulator Source and Multi-Wavelength Anomalous Dispersion: A Proposed Program for the National Synchrotron Light Source

\section{Preamble}

High-resolution $x$-ray crystallography has had unparalleled impact on the course of biological and biomedical research. Structural information is providing exquisite detail about the assembly and control of macromolecular complexes and the mechanisms of macromolecular function. Future advances in biological and biomedical science require an ever-expanding structural database to keep pace with the rapidly growing body of biochemical and genetic data. Given the unprecedented volume of data accruing from the genome sequencing projects, there is no period in history that will benefit as much from an expansion of structural resources and methodologies. This core project is directly taking up this challenge by expanding the national and worldwide MAD data collection facilities and in developing new technologies for efficient and rapid de novo structure solution by MAD crystallography.

\section{Overview \& Significance}

Synchrotron radiation is the single most valuable tool for solving the 'phase problem', which remains one of the rate limiting steps in de novo macromolecular structure determination by $X$-ray crystallography. The broad band nature of bending magnet and wiggler synchrotron radiation sources allows for data collection at, and around, the absorption edges of anomalous scatterers maximizing the anomalous contribution of each derivative. The ability to collect data at and around the absorption edges of anomalous scatterers allows maximal extraction of phase information from each individual derivative by the optimization of the anomalous scattering signal (Hendrickson, 1991). Thus, the incorporation of only a single type of anomalous scatterer allows for highly efficient structure determination by multi-wavelength anomalous dispersion (MAD) techniques. Optimization of anomalous signal also significantly enhances the quality of maps calculated by traditional isomorphous replacement methods. In many cases, the high brightness of synchrotron $x$-radiation beamlines provide a significant enhancement in the resolution of data collected from both weakly diffracting and small crystals relative to laboratory-based rotating anode generators. In fact, many recent problems involving macromolecular assemblies, with weakly diffracting crystals, provide data inadequate to fully appreciate reaction mechanism even when examined on focused bending magnet sources. Insertion devices will play an increasing role in solving these difficult crystallographic problems. However, despite the expanding role the MAD method plays in protein crystallography there are surprisingly few beamlines (and fewer insertion device lines) available for such experiments. Consequently, users often had to wait for extended periods of time in order to perform a MAD experiment. In addition, there is a regional need for such devices to minimize travel time and expense for investigators. 
Both the brightness and tunability of synchrotron sources contributes significantly to their value to structural biology investigations, however extremes of brightness and tunability are in some respects mutually exclusive. For example, sagitally focused bending magnet sources (like X4A at NSLS) have wide ranges of tunability and moderate brightness (NSLS, 1997). Undulator sources (like the Structural Biology line at APS) have extreme brightness, but the spectral width is narrow. However, changes in the undulator parameters (e.g. "the gap") can be made in order to modulate the frequency. For example, a fully tunable undulator with a $3.3 \mathrm{~cm}$ period on the APS (undulator A) will deliver $4-13 \mathrm{keV}$ in the first harmonic (Shenoy et. al., 1988).

Wiggler sources (like $\times 25$ at NSLS and F2 at CHESS) provide an intermediate solution, with high brightness, broadband radiation, and high critical energies. At the NSLS, the potential for insertion devices exists only at the straight sections, beamlines $X[4 n+1]$, where $n=0$ to 7 (see below). The only two remaining straight sections currently uncommitted to insertion devices are $\times 9$ and $\times 29(n=2,7)$. Unfortunately, the required pairs of radio frequency (rf) cavities in these locations precludes incorporation of a conventional wiggler design for these beamlines.

The NSLS, in collaboration with SPring-8, Japan, has designed, built and tested a prototype "small-gap" undulator, whose compactness allows it to reside between the rf cavities that exist at $X 9$ and $\times 29$. This prototype in-vacuum undulator (IVUN), with a period of $11 \mathrm{~mm}$, a minimum useful gap of $3.3 \mathrm{~mm}$ (maximum of $10 \mathrm{~mm}$ ) and a magnetic field of $0.678 \mathrm{~T}$ is currently installed in the straight section for beamline $\times 13$. These parameters lead to a measured fundamental at $4.6 \mathrm{keV}$, second harmonic at $8.7 \mathrm{keV}$, and a third harmonic at $13.9 \mathrm{keV}$ at the current ring energy of $-2.5 \mathrm{GeV}$. The brightness of IVUN is comparable (slightly higher) than the $\times 25$ wiggler. The successful demonstration of IVUN as a device capable of MAD studies would lay the foundation for expansion of undulator MAD at the NSLS through future development of the $\times 13, \times 29$ or $\times 9$ stations.

Biological Impact. Specific structural projects that will benefit from this facility, due to weakly diffracting crystals or difficulty in obtaining multiple isomorphous derivatives, include the accessory proteins regulating eukaryotic RNA polymerase, components of the eukaryotic DNA polymerase system, proteins that crosslink, bundle and sever actin filaments, SH2 and SH3 modules, phosphatases, restriction enzymes, cyclin-dependent kinases, immune receptors and growth factors. These structural results will have major impact on areas of central importance in cell biology, including transcriptional control of gene regulation, signal transduction pathways which link external stimuli to intracellular responses, eukaryotic DNA replication, regulation of the actin cytoskeleton which is involved in cell motility, division and morphology, the immune response and cell cycle control. These structural results will also directly benefit the programs of our immediate collaborators, and, most importantly will provide the structural foundation that is essential for future work by the large number of biologists working in each of these sub-disciplines. The proposed work covers nearly every major field on modern molecular and cellular biology, and will provide fundamental mechanistic information about normal physiology and pathogenic processes such the initiation of transformation, tumor progression and metastasis. Several detailed examples of this impact of such a device are provided below.

Task Force Recommendations. Recently a task force to examine the issue of the technical capabilities and biological impact of a small-gap undulator device was convened by $\mathrm{Dr}$. Michael Hart, Chairman of the NSLS. A Structural Biology sub-group, consisting of Dr. 
Mark Chance, Albert Einstein College of Medicine, Dr. Stephen Burley, Rockefeller University, and Dr. Wayne Hendrickson, Columbia University provided a report to the Chair of the committee, Dr. Jerry Hastings, NSLS. A summary of their report is described below:

The development of the small-gap undulator (SGU) device makes available two new straight sections that were previously considered unusable for insertion devices. The growth of protein crystallography programs, and the need for high brightness radiation tunable at the edges of anomalous scatterers suggests that the development of a specific SGU device for protein crystallography applications is warranted.

From a protein crystallography perspective, the elements for MAD that match $x$-ray undulator characteristics under discussion include the following:

\begin{tabular}{lc} 
Element & Edge (KeV) \\
\hline $\mathrm{Br}-\mathrm{K}$ & 13.5 \\
$\mathrm{~Pb}-\mathrm{L}$ & 13.0 \\
$\mathrm{Se}-\mathrm{K}$ & 12.6 \\
$\mathrm{Hg}-\mathrm{L}$ & 12.3 \\
$\mathrm{Au}-\mathrm{L}$ & 11.9 \\
$\mathrm{Pt}-\mathrm{L}$ & 11.6 \\
$\mathrm{Xe-L}(I I I)$ & 4.8 \\
$\mathrm{I}-\mathrm{L}$ (III) & 4.6 \\
$\mathrm{U}-\mathrm{M}(\mathrm{V})$ & 3.5
\end{tabular}

We also established that a typical MAD experiment should range from -100 to +300 ev with respect to these absorption edges. It also may be desirable to move above this to $+1 \mathrm{kev}$ if possible.

In the recent calculation provided by Peter Stephan, a device optimized at $\sim 2.5 \mathrm{GeV}$ for the $\mathrm{Hg}$ and $\mathrm{Se}$ edges at the 3rd harmonic is feasible. It would also allow tuning through the $I$ and $X e$ edges at the first harmonic, although with substantial flux changes. I have annotated this curve, indicating the "gap" for each energy point. The "boxes" adjacent to each element represent the MAD tuning range for that element, with the vertical lines representing, $-100,0,+300$, and $+1 \mathrm{KeV}$.

In discussions with Peter Stephan, a device optimized for $2.8 \mathrm{GeV}$ would have very similar tuning curves. Thus, a question arises, which ring energy does one choose for optimizing the undulator design. Under current operating conditions, either choice of ring energy would allow MAD experiments only for $50 \%$ of the time. In essence, the undulator should be a motivation to freeze the energy. Peter Stephan informs me that a "refit" of the undulator to accommodate a new ring energy would require an additional investment of at least $20 \%$ of the cost of the device.

The current availability of the $\times 29$ port makes it a reasonable choice for siting a protein crystallography undulator device. A number of beamline designs could also be accommodated at the $\times 29$ station based on preliminary estimates by Mr. Michael Sullivan. 
This would require that some optical elements be located inside the shield wall. If failures of these elements occur, some provisions for emergency ring access to provide repairs would need to be agreed upon.

Additional issues include: 1) PRT membership, 2) funding and 3) the current use of all straight sections around the ring.

1) The committee will require more time and information in order to recommend any specific PRT membership. Many groups are likely to be interested including The New York Crystallography Consortium, Howard Hughes Medical Institute, NIH and others. The New York group (AECOM, Rockefeller, SKI) has funding in hand to establish such a program and could easily move from the X9A station once the undulator is in place.

2) Another issue concerns the funding of the project and the provision for general user time. We discussed at the meeting that the total cost of building and installing the front end to the NSLS was likely in the range of 1-1.5 million dollars and might be completed by the December 1999 shutdown given sufficient advance planning. The cost of the beamline and detectors is similar at 1-1.5 million dollars. The cost of operating the facility over five years is likely close to 1.5 million dollars.

The total cost of the project over five years is 3.5 to 4.5 million, the PRT share of the costs would be $65-70 \%$, neglecting the cost of operating the ring bourne entirely by NSLS. To provide a meaningful program for the PRT, a minimum of $60 \%$ of the time would be required for the PRT, while $75 \%$ of the time would be required for a large PRT group.

3) The significant demand for insertion device beam time and the relative position of the NSLS Structural Biology Program vis a vis ALS, SSRL and APS suggests that one additional station may not be nearly sufficient to meet the demand. Thus, the utilization of other stations must be investigated.

\section{Summary:}

1) Build and install SEUN (Selenium Undulator) at $X 29$ with the front end paid for by NSLS by January 1,2000. Optimize the device for either -2.5 or $2.8 \mathrm{GeV}$ depending on long term ring plans. Freeze the ring energy at this valve.

2) Investigate the designation of other straight sections for protein crystallography, such as $X 13$ or $\times 21$, to provide an appropriate balance for this activity with respect to insertion device availability.

3) Investigate the desire of the appropriate stakeholders to participate in insertion device programs for crystallography to provide funding for the beamline and for the user program.

\section{References}

Hendrickson, W.A., (1991) Detection of Macromolecular Structure from Anomalous Diffraction with Synchrotron Radiation. Science, 254: 51-58 NSLS, "National Synchrotron Light Source 1996 Activity Report. 


\section{Biological Impact of Small-Gap Undulator}

MAD Structural Studies of the Actin Based Cytoskeleton using a Small-Gap Undulator Dr. Steven C. Almo, Albert Einstein College of Medicine

Research being carried out in Dr. Almo's laboratory is focused on the atomic-level mechanisms which regulate the actin cytoskeleton. The actin cytoskeleton is involved in mediating fundamental cellular processes including motility, cytokinesis, muscle contraction, endocytosis, intracellular transport and the establishment and maintenance of cell morphology. The laboratory is working towards an enhanced structural and functional understanding of 1 ) the proteins which regulate the equilibrium between monomeric (G-) and filamentous (F-) actin, 2) the proteins which regulate the length distribution of individual actin filaments and 3 ) the proteins responsible for bundling filaments into numerous types of higher order aggregates which direct diverse biochemical processes. Synchrotron radiation is playing an increasingly important role in these projects due to limitations in resolution and difficulty in obtaining multiple isomorphous derivatives. This structural information is providing the basis for extended structure-function analysis involving in vitro biochemical characterization of mutants, in vivo expression of mutant proteins with defined biochemical activities and correlation with electron microscopic studies.

We have an established program (funded by the National Institutes of Health) directed towards structural analysis of the profilins, a group of small proteins (MW - 12-15kD) which are responsible for the regulation of filamentous actin structures in all eukaryotic cells. We have solved profilin structures from a number of sources, including two isoforms from Acanthamoeba (Fedorov, et al., 1994), plants (Fedorov, et al., 1997a,b), $S$. cerevisiae (Eads, et al., submitted) and humans. These studies have provided structural information about the evolutionary development of this protein family and the mechanism by which profilins are regulated by polyphosphoinosotides (e.g., PIP2). This regulation indicates that profilin may serve to couple the re-organization of the actin cytoskeleton to phosphoinositide metabolic and signal transduction pathways. We have also recently solved the structure of human profilin bound to an oligo-proline peptide (Mahoney, Janmey and Almo, submitted), which describes a mechanism for the intracellular localization of profilin to sites requiring extensive actin polymerization.

Cofilin is a ubiquitous eukaryotic protein which severs actin filaments in vitro and is responsible for the dynamics of actin filaments in vivo. In collaboration with David Drubin (University of California, Berkeley), we have solved the structure of cofilin from $S$. cerevisiae to $2.3 \AA$ resolution (Fedorov, et al., 1997c). This structural data has allowed for the design of mutations, which have been tested in vitro for F-actin binding and depolymerizing activity. In vivo expression of these mutants has allowed for the actin binding surfaces of yeast cofilin to be defined based on classical phenotypic analysis (Lapalainen, et al., 1997). We have also prepared crystals of severin domain-2 in the absence of calcium. Severin is an F-actin binding and severing protein which is positively regulated by calcium. We have been unable to solve the structure using the NMR model of the calcium bound form as the search model for molecular replacement and despite extensive efforts, we have only found a single suitable mercury derivative. The structure of the calcium free form will complete the atom-level mechanism for activation of F-actin binding and actin severing. 
A new program in the laboratory (funded by the National institutes of Health) is directed towards the structural characterization of the proteins which crosslink individual actin filaments into higher order bundles and aggregates. In collaboration with Paul Matsudaira (MIT), we have recently solved the structure of the amino terminal actin binding domain from human fimbrin (Goldsmith, et al., 1997a,b), which represents the first structure of a domain involved in actin cross-linking. The solution of this structure was aided by the collection of isomorphous and anomalous data from a mercury derivative at the absorption edge at beam line 1-5AD at SSRL. Based on this structure, we have proposed a detailed mechanism for binding to F-actin which we are now testing by in vitro and in vivo characterization of specific mutants. Furthermore, in collaboration with David DeRosier (Brandeis University) we have docked the fimbrin structure into a low resolution $(-25 \AA)$ helical reconstruction of $\mathrm{F}$-actin decorated with the actin binding domain. This provides the first detailed model of an assembly between F-actin and an actin crosslinking protein. The structure solution of fimbrin was hampered by difficulty in obtaining multiple isomorphous derivatives; in many cases isomorphism extended to only $6 \AA$ or less. The solution of this structure would have been greatly facilitated by the ability to collect MAD data at the selenium edge.

We have recently begun structural characterization of human fascin $(55 \mathrm{kD})$, an unrelated actin crosslinking protein which is regulated by phosphorylation. This has proven to be a challenging crystallographic problem, as these crystals only diffract to $\sim 3.5-4.0 \AA$ on a rotating anode generator. Furthermore, despite over 100 attempts, an isomorhpous derivative has only been obtained by co-crystallization with $A u(C N) 2$. Other potential derivatives suffer from extensive changes in unit cell dimensions and severe nonisomorphism. The search for heavy atoms is further complicated by the requirement for high concentrations of reducing agent (i.e., $20 \mathrm{mM}$ DTT) to maintain crystal integrity. These crystals diffract to $2.8 \AA$ at $F 2$ at CHESS, showing that an intense $X$-ray source will be required for the successful completion of this structure. Additionally, flash cooling introduces extensive variability in unit cell parameters of both native and derivative crystals, indicating that structure solution from a single crystal will be optimal. Thus fascin represents a case in which MAD data collection at the selenium edge using the high intensity provided by an insertion device provides the most direct route to successful structure solution. The fascin structure will be particularly informative, as it will show the proximity of the two independent F-actin binding sites to each other and to the site of phosphorylation and will allow for a correlation with extensive electron microscopic data describing the actin-fascin bundle.

Finally, we have produced crystals of ABP-30, a novel actin binding protein from Dictyostelium which is unique amongst the crosslinking proteins in that it contains three distinct F-actin binding sites. These crystals diffract only very weakly to $6 \AA \AA$ on a laboratory-rotating anode, but the resolution can be extended to better than $3.5 \AA$ resolution using the radiation provided by the bending magnet at X9B at NSLS. The weak diffraction excludes the possibility of searching for heavy atoms in the laboratory, and suggests that successful structure solution will absolutely require the use of MAD data collection at the selenium edge with the high flux available from a small gap undulator source.

Rationale for MAD Undulator. The current crystallographic projects under investigation in our lab highlight the requirement for an undulator source which can be tuned around the 
selenium edge. In many cases MAD data collection from a single crystal provides the only way the overcome difficulties related to non-isomorphism which are associated with the preparation of standard heavy atom derivatives and cryogenic data collection. The biosynthetic incorporation of seleno-methionine allows for structure solution from a single crystal. In cases of weakly diffracting crystals, the use laboratory and bending magnet sources does not afford the opportunity to obtain the required resolution, nor to efficiently screen for isomorphous derivatives. Only the combination of high flux and tunability at the selenium edge, which can be provided by the third harmonic from the described undulator, will allow for the structure solution of these most challenging problems which are at the heart of cell biology.

\section{References}

Eads, J.C., Mahoney, N.M., Vorobiev, S., Haarer, B.K. and Almo, S,C. Structure Determination and Characterization of Saccharomyces cerevisiae profilin. Submitted.

Fedorov, A.A., Magnus, K.A., Graupe, H., Latman, E.E., Pollard, T.D. and Almo, S.C. "X-ray Structures of Isoforms of the Actin Binding Protein Profilin that Differ in Their Affinity for Polyphosphoinositides" Proc. Natl. Acad. Sci., USA 91, 8636-8640 (1994).

Federov, A.A., Ball, T., Valenta, R. and Almo, S.C. "X-ray Crystal Structure of Birch Pollen Profilin and Phl p 2". Int. Arch. Allergy Immunol 113, 109-113 (1997a).

Fedorov, A.A., Ball, T., Mahoney, N., Valenta, R., Almo, S.C. "Crystal Structure and IgE-epitope Mapping of Birch Pollen Profilin: Molecular Basis for Allergen CrossReactivity. Structure, 5, 33-45 (1997b).

Fedorov, A.A., Lapalainen, P., Federov, E., Drubin, D. and Almo, S.C. "The crystal structure of Yeast Cofilin", Nature Structural Biology, 4,366-369 (1997c).

Goldsmith, S., Pokala, N., Matsudaira, P. and Almo, S.C. "Crystallization and Preliminary Crystallographic Characterization of the $\mathrm{N}$-terminal Actin binding Domain of Human Fimbrin" Proteins", 28, 452-3 (1997a).

Goldsmith, S., Pokala, N., Shen, W., Federov, A.A., Matsudaira, P. and Almo, S.C. "The Structure of an Actin-crosslinking Domain from Human Fimbrin", Nature Structural Biology, 4, 708-712 (1997b).

Lapalainen, L., Fedorov, A.A., Federov, L., Almo, S.C. and Drubin, D.G. Mapping the Actin Binding Sites on Cofilin through genetic interaction in yeast, EMBO J., in press. \{1997\}

Mahoney, N.M., Janmey, P.A. and Almo, S.C. A Novel Proline-Rich Ligand Binding Motif Involved in Morphogenesis and Cytoskeletal Regulation: Crystal Structure of the Profilin-Poly-L-Proline Complex. Submitted. 


\section{Structural Analysis of Restriction Enzymes: MAD Studies Using a Small-Gap Undulator}

\section{Dr. Aneel Aggarwal, Mt. Sinai School of Medicine}

The research in our laboratory represents a consistent effort to understand how proteins bind DNA, leading to specific DNA cleavage and the regulation of transcription. Our efforts are concentrated on two categories of DNA binding proteins, namely restriction enzymes and transcription factors. Synchrotron radiation, in the form of the multiwavelength anomalous diffraction (MAD) method, is an essential technique for the success of our research program. The measurement of multi-wavelength data near the $\mathrm{Se}$ and $\mathrm{Br}$ $\mathrm{K}$-edge absorption profiles has led to the determination of three structures and will be the basis of most of our future synchrotron data collection efforts. However, despite the expanding role the MAD method plays in protein crystallography there are surprisingly few beamlines dedicated for such experiments. Consequently, we have often had to wait for extended periods of time in order to perform a MAD experiment. The development of $\times 13$ as a bright MAD beamline provides a unique opportunity to alleviate delays in acquiring beamtime and in enhancing the overall effectiveness of our research.

\section{A. Restriction Enzymes.}

Type II restriction endonucleases are ideal systems for studying selectivity, due to their high specificity and striking variety [1]. The enzymes recognize and cleave DNA sequences that vary between four to eight base pairs. Their specificity is remarkable. A single base pair change within the recognition sequence can lead to well over a million-fold reduction in activity. An understanding of sequence specific cleavage is relevant to proteins mediating site-specific recombination and DNA repair by excision. Our work is focused on endonucleases Sfil, BamHI, and Fokl.

Sfn. The Sfi endonuclease cleaves DNA by a mechanism that differs from other restriction enzymes. Sfil exists in solution as a tetramer and it appears to interact with two copies of its 8-bp recognition sequence. The two sites can be either on the same or different DNA molecules in order to activate the enzyme for cleavage [2]. The Sfi reaction has marked similarities to site-specific recombinases and provides a system for understanding long-range interactions on DNA. We have obtained Sfi tetramer/DNA cocrystals that diffract to at least $1.9 \AA$ in resolution and are stable in the $X$-ray beam upon freezing. Because Sfn bears little sequence similarity to other endonucleases, its structure will be determined by a combination of MAD and multiple isomorphous replacement (MIR) methods. For MAD phasing, the Sfil monomer (270 amino acids) contains 4 methionines that could be substituted by selenomethionines and the DNA oligomer contains 11 thymines that could be substituted by bromouracils. For the measurement of MAD data, the absorption K-edges of both $\mathrm{Se}$ and $\mathrm{Br}$ will be accessible at beamline $X 13$.

BamHI. We have determined the high-resolution structures of both the "free" and specificDNA bound forms of the enzyme [3-5]. These structures represent the first step in the catalysis of DNA. The free BamHI structure was solved by the MAD method using a selenomethionine labeled protein [4]. Our plan is now to determine high-resolution structures at other stages of the BamHI catalytic pathway. We have cocrystallized BamHI

with non-cleavable DNA analogs in the presence of $\mathrm{Mg}^{2}+$ and $\mathrm{Mn}^{2}+$ ions, and initiated efforts to cocrystallize BamHI with non-specific DNA sites. Together, these structures will 
provide a complete, dynamic view of the sequence of events underlying sequence specific cleavage. As a supplement to the application of molecular replacement (MR) methods, the availability of selenomethionine labeled $B a m H I$ makes it possible to measure MAD data on these crystals.

Fokl. Fokl is a member of a novel class of bipartite restriction enzymes that recognize an asymmetric DNA sequence and cleave DNA a short distance away from that sequence. Because of its unusual bipartite nature, Fokl has been used to create artificial enzymes with new specificities [6]. We have determined the crystal structure of the complete Fokl enzyme (578 amino acids) bound to DNA at $2.8 \AA$ resolution [7]. As anticipated, the enzyme contains $\mathrm{N}$ - and $\mathrm{C}$-terminal domains corresponding to the DNA recognition and cleavage functions, respectively. However, the cleavage domain is found to be sequestered in a piggyback fashion by the recognition domain, suggesting a novel mechanism of activation in which the cleavage domain swings over to the major groove for cleavage. To observe this "open" configuration, we are cocrystallizing Fokl with longer DNA fragments under conditions favoring the dissociation of the two subdomains. Extensions of this work may require the use of MAD method in the future.

\section{B. Transcription Factors}

The work on eukaryotic transcription factors is aimed at understanding how these proteins recognize their DNA sites and exert their activating or repressing influence on transcription. Understanding how these factors work is one of the major challenges in biology today. It is central to understanding how organisms develop, respond to extracellular signals, and acquire specific tissues.

Pituitary Specific Transcription Factor Pit-1. Pit-1 is expressed exclusively in the anterior pituitary gland and is responsible for the activation of several genes, including the prolactin, the growth hormone, and the thyroid stimulating hormone genes [8]. Pit-1 is a founder member of the POU domain class of transcription factors. Many of the mutations causing combined pituitary hormone deficiency (CPHD) in humans have been mapped to the Pit-1 POU domain. The disease leads to combined deficiencies in prolactin, growth hormone, and the thyroid stimulating hormone, with the patients often being characterized by physical and mental retardation [9]. We have determined the structure of Pit-1 POU domain bound to DNA as a homodimer [10]. The structure was solved by the MAD method using data measured near the $\mathrm{Br}$ K-edge. The structure suggests an intriguing transcriptional model, in which Pit-1 interacts with distinct coactivators when bound to different DNA response elements. To explore this, we have begun work to isolate putative coactivators using pituitary whole cell extracts and the yeast two-hybrid system (in collaboration with Dr. Rosenfeld's laboratory at UCSD). We have purified a potential cofactor and initiated studies to characterize it biochemically and to cocrystallize it with Pit-1. The experience we have gained in measuring Br MAD data will be directly applicable to the Pit-1/coactivator complexes.

Interferon Regulatory Factor IRF-1. The regulation of the human interferon-b (IFN-b) gene presents one of the best characterized system for understanding inducible transcription [11]. The gene is activated in response to viral infection, and the 50-bp regulatory element is located immediately upstream of the transcription start site. A crucial factor in the induction of the gene is the interferon regulatory factor-1 (IRF-1), which also manifests tumor-suppression properties [12]. We have determined the structure of IRF-1 DNA 
binding domain bound to an oligomer from the IFN-b regulatory element [13]. To solve the structure, we again took advantage of bromouracils substitutions within the DNA fragment to perform a MAD experiment. Efforts are now underway to prepare crystals of IRF-1/DNA complex in association with other protein components involved in IFN-b regulation, including the transcription factors NF-kB and HMG I(Y).

Developmental Homeodomains. The homeodomain is one of the most common DNA binding motifs found in developmental regulators [14]. A central issue in development is how different homeodomain proteins achieve their specificities in vivo. Distantly related homeodomains often bind to similar DNA sites, and yet in cells or tissue they display remarkable biological specificity. An important recent advance has been the identification of a cofactor, Extradenticle (Exd), that can modulate the DNA binding specificities of some but not other homeodomains [15]. To understand how this is achieved, we have initiated studies to cocrystallize Exd with the homeotic protein Ultrabithorax (Ubx). Because these are bacterially expressed proteins, it would be relatively straightforward to prepare selenomethionine labeled variants for structure determination by the MAD method.

Rationale for MAD Undulator. All of our MAD experiments have been performed either at the $\mathrm{Se}$ or $\mathrm{Br} \mathrm{K}$-edge absorption profiles. Protein-DNA complexes allow the incorporation of the two atoms in a straightforward manner. The ability to collect MAD data at the Se and $\mathrm{Br}$ absorption edges will have a major impact on our work on protein-DNA interactions.

\section{Literature Cited}

1. Roberts, R.J., and S.E. Halford (1993) Type II restriction endonucleases, in Nucleases (S.M. Linn, R.S. Lloyd, and R.J. Roberts, Eds.) Cold Spring Harbor: New York.

2. Wentzell, L.M., T.J. Nobbs, and S.E. Halford (1995). The Sfil restriction endonuclease makes a four-stranded DNA break at two copies of its recognition sequence. J. Mol. Biol. 248, 581-595

3. Newman, M., T. Strzelecka, L.F. Dorner, I. Schildkraut, and A.K. Aggarwal (1994). Structure of restriction endonuclease BamHI and its relationship to EcoRI. Nature 368, 660-664

4. Newman, M., T. Strzelecka, L.F. Dorner, I. Schildkraut, and A.K. Aggarwal (1994). Structure of restriction endonuclease BamHI phased at 1.95 A resolution by MAD analysis. Structure 2, 439-452

5. Newman, M., T. Strzelecka, L. Dorner, I. Schildkraut, and A.K. Aggarwal (1995). Structure of BamHI endonuclease bound to DNA: partial folding and unfolding on DNA binding. Science 269, 656-663

6. Kim, Y.-G., J. Cha, and S. Chandrasegaran (1996). Hybrid restriction enzymes: zinc finger fusions to Fokl cleavage domain. Proc. Natl. Acad. Sci. 93, $1156-1160$

7. Wah, D.A., J.A. Hirsch, L.F. Dorner, I. Schildkraut, and A.K. Aggarwal (1997). Structure of the multimodular endonuclease Fokl bound to DNA. Nature $388,97-100$ 
8. Rhodes, S.J., G.E. DiMattia, and M.G. Rosenfeld (1994). Transcriptional mechanisms in anterior pituitary cell differentiation. Curr. Opin. Cell Biol. 4, 709717

9. Ohta, K., Y. Nobukuni, H. Mitsubuchi, S. Fujimoto, N. Matsuo, H. Ingaki, F. Endo, and I. Matsuda (1992). Mutations in the Pit-1 gene in children with combined pituitary hormone deficiency. Biochem. Biophys. Res. Comm. 189, $851-855$

10. Jacobson, E.M., P. Li, A. Leon-del-Rio, M.G. Rosenfeld, and A.K. Aggarwal (1997). Structure of Pit-1 POU domain bound to DNA as a dimer: unexpected arrangement and flexibility. Genes \& Dev. 11, 198-212

11. Maniatis, T., L.-A. Whittemore, W. Du, C.-M. Fan, A.D. Keller, V.J.

Palombella, and D.N. Thanos (1992). Positive and negative control of human interferon-b gene expression in Transcriptional Regulation (S. McKnight and K. Yamamoto, Eds) Cold Spring Harbor Laboratory Press: New York. p. 1193-1220.

12. Taniguchi, T., H. Harada, and M. Lamphier (1995). Regulation of the interferon system and cell growth by the IRF transcription factors. J. Cancer Res. Clin. Oncol. 121, 516-520

13. Escalante, C.R., J. Yie, D. Thanos, and A.K. Aggarwal (1997). DNA recognition underlying interferon regulation: Structure of IRF-1 bound to the PRD I element. Nature, in press.

14. Gehring, W.J., M. Affolter, and T. Burglin (1994). Homeodomain Proteins. Annual Review of Biochemistry 63, 487-526

15. Mann, R.S., and C.K. Chan (1996). Extra specificity from Extradenticle: the partnership between Hox and Pbx/Exd homodomain proteins. Trends Genet. 12 , 258-262. 
MAD Studies of Eucaryotic Transcription Factors using a Small-Gap Undulator

\section{Dr. Stephen Burley, Rockefeller University}

Research being carried out in Dr. Burley's laboratory is primarily concerned with atomiclevel elucidation of the mechanisms by which gene expression is controlled in eukaryotes. The laboratory is working towards improved structural and functional characterizations of the general transcription factors and associated regulatory proteins that together with RNA polymerase II (pol II) mediate appropriate nuclear responses to incoming developmental and environmental signals (reviewed in Nikolov \& Burley, 1997). Crystallographic analyses using synchrotron radiation have played a critical role in this work. Multiple isomorphous replacement (MIR), molecular replacement (MR) and multi-wavelength anomalous dispersion (MAD) methods are being used routinely in structure determinations. However, cryogenic studies often introduce non-isomorphism emphasizing the need for MAD sources.

Structural and functional studies supported by the Howard Hughes Medical Institute are focused on the general transcription machinery that controls initiation of messenger RNA production by pol II. In the most general case, this process begins with transcription factor (TF) IID recognition of the TATA element, found about 30 base pairs (bp) upstream of the transcription start site. This process is supported by the TATA box-binding protein subunit of TFIID, known as TBP, which is conserved across evolution (i.e., between archaebacteria and human). Our studies of this protein have yielded structures of the apo-protein (Nikolov et al., 1992) and its complex with the TATA box (Kim et. al., 1993), the latter refined to $1.9 \AA$ resolution with data collected on beamline $X 25$, NSLS (Kim \& Burley, 1994). Other work on TFIID includes a MAD structure determination of another two of its subunits that proved to be structural homologues of histones $\mathrm{H} 3$ and $\mathrm{H} 4$ (Xie et. al., 1996), using beamline X4A, NSLS. Most recently, we obtained a co-crystal structure of a triple complex of TFIIB, TBP and DNA (Nikolov et al., 1995) that allowed us to visualize the second assembly step en route to a fully functional transcription complex also known as a pre-initiation complex or PIC. These structural studies have revealed a novel mechanism of DNA recognition by TBP, involving intercalation, unwinding and bending. In addition, they have demonstrated a direct structural connection between the pol 11 transcriptional apparatus and the DNA packaging machinery responsible for condensation of DNA into chromatin. Finally, they have provided critical mechanistic insights into the first two steps of transcription initiation by pol II in euakaryotes, which are heavily regulated.

The laboratory is also involved in structural studies of members of the basic/helix-loophelix/leucine zipper family of transcriptional regulators (supported by the National Institutes of Health). These proteins recognize promoter proximal elements found anywhere between 50 and 200 bp upstream of the transcription start site, where they form stable protein-DNA complexes that stimulate the activity of the PIC. To date, we have determined three co-crystal structures, including Max, upstream stimulatory factor (USF) and sterol response element binding protein 1 (SREBP-1). The structure of the Max homodimer bound to 5'CACGTG3' was determined via MIR with iodinated DNA derivatives at $2.9 \AA$ resolution, using data collected at beamline F1, CHESS (Ferre-D'Amare et al., 1993). The structure of the USF homodimer bound to $5^{\prime}$ CACGTG3' was determined via molecular replacement at $2.9 \AA$ resolution, using data collected at beamline F1, CHESS and 
beamline X25, NSLS (Ferre-D'Amare et al., 1994). The structure of the SREBP-1 homodimer bound to a sterol response element was determined via MIR with iodinated DNA derivatives and MR with the Max homodimer structure at $2.3 \AA$ resolution, using data collected at beamline F1, CHESS (to be published). The SREBP-1 structure was particularly challenging due to the large unit cell (space group P6122; $a=b=94.6, c=459.1$ ), and absolutely required the use of an insertion device. The Max homodimer co-crystal structure was the first member of this biologically-important family of transcriptional regulatory proteins to be visualized at near atomic resolution. Taken together, these structures revealed a DNA-directed induced fit mechanism of recognition. The basic region undergoes a random coil to helix folding transition on interaction with DNA. In addition, each structure made a considerable impact on studies of their biology.

Rationale for MAD Undulator. The research program described here will increasingly rely on bright MAD sources for a number of reasons. First, access to very bright radiation has enabled structure determinations to proceed in situations where the crystal quality was insufficient for conventional X-ray analysis. Second, and increasingly important, the utilization of cryogenic data collection has introduced a much greater level of nonisomorphism between crystals of a particular protein. While cryogenics is essential for preserving the diffraction of most of the crystals the laboratory has worked on, its utilization has made it much more difficult to obtain accurate phases using conventional MIR techniques. MAD analysis has therefore been utilized to get around this problem. In addition, sufficient heavy atom derivatives are not always obtainable, in these cases SeMet labeled protein and/or Br labeled nucleic acids must be employed (Xie et al., 1996). Lastly, the increasing interest in larger proteins and larger macromolecular complexes presumes weaker diffracting, small, and fewer crystals will be available. Thus, the increased source brightness provided by the small-gap undulator compared to focused bending magnet lines will be of significant benefit.

\section{References}

Ferre-D'Amare, A.R., G.C. Prendergast, E.B. Ziff, and S.K. Burley (1993), Recognition by Max of its cognate DNA through a dimeric b/HLH/Z domain, Nature $363,38-45$.

Ferre-D'Amare, A.R., P. Pognonec, R.G. Roeder, and S.K. Burley (1994), Structure and function of the $\mathrm{b} / \mathrm{HLH} / \mathrm{Z}$ domain of USF, EMBO J. 13, 180-189.

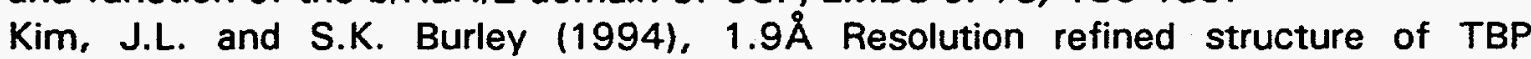
recognizing the minor groove of TATAAAAG, Nature Structural Biology 1, 638653.

Kim, J.L., D.B. Nikolov, and S.K. Burley (1993), Co-crystal structure of TBP recognizing the minor groove of a TATA element, Nature 365, 520-527.

Nikolov, D.B, H. Chen, E.D. Halay, A. Usheva, K. Hisatake, D.K. Lee, R.G. Roeder, and S. K. Burley (1995), Crystal structure of a TFIIB-TBP-TATA element ternary complex, Nature 377, 119-128.

Nikolov, D.B. and S. K. Burley (1997), RNA polymerase II transcription initiation: A structural view, Proceedings of the National Academy of Sciences USA 94, 15-22. 
Nikolov, D.B., S.-H. Hu, J.P. Lin, A. Gasch, A. Hoffmann, M. Horikoshi, N.-H. Chua, R.G. Roeder, and S. K. Burley (1992), Crystal structure of TFIID TATA-box binding protein, Nature 360, 40-46.

Xie, X. S.L. Cohen, U. Mirza, B.T. Chait, A. Hoffmann, R.G. Roeder, Y. Nakatani and S. K. Burley (1996), Structural similarity between TAFs and the heterotetrameric core of the histone octamer, Nature 380, 316-322. 
MAD Structural Studies of Proteins Involved in Cellular Signal Transduction and DNA Replication using a Small-Gap Undulator

\section{Dr. John Kuriyan, Rockefeller University}

Research being carried out in Dr. Kuriyan's laboratory is concerned mainly with the atomiclevel mechanisms of intra-cellular signal transduction and chromosomal DNA replication. Crystallographic analysis using synchrotron radiation has played a critical role in both projects, particularly due to the application of multi-wavelength anomalous diffraction (MAD).

The laboratory is working towards an increased understanding of the kinases, phosphatases and associated regulatory proteins that interact to produce appropriate cellular responses to incoming signals. A particular focus of interest is the mechanism by which the cytoplasmic Src-family tyrosine kinases are regulated. First identified as the oncogene product of the Rous sarcoma virus, the Src tyrosine kinase and its relatives play key roles in signaling pathways that are involved in inter-cellular communication and the regulation of growth. In addition to the catalytic kinase domain, these proteins contain two modular units, the Src homology-2 and 3 (SH2 and $\mathrm{SH} 3)$ modules that regulate the activity of the enzyme and control its interactions with other proteins. The laboratory has recently made two advances in understanding the regulation of Src-family tyrosine kinases. The structural basis for the tight interaction of the HIV-1 Nef protein with certain Src-family tyrosine kinases has been mapped, and the crystal structure of a down-regulated form of a Src-family tyrosine kinase, including the regulatory and domains, has been determined.

Nef is a gene product of human and simian immunodeficiency viruses (HIV-1, HIV-2, SIV) that is critical for the development of AIDS. Nef contains a highly conserved "PxxP" sequence motif, that is the signature of $\mathrm{SH} 3$ binding proteins, and Nef binds tightly to certain Src-family SH3 domains. Mutagenesis of Nef showed that the PxxP motif is essential for optimal spread of HIV-1 virus in primary cell cultures, suggesting that the virus has evolved to exploit SH3-mediated interactions with cellular proteins to somehow enhance its replication. The crystal structure of the conserved core of HIV-1 Nef has been determined, in complex with the SH3 domain (R96I mutant) of the Src family tyrosine kinase Fyn (Lee et al., 1996). The analysis, carried out at beamline X4A at NSLS, Brookhaven, and at CHESS, relied on a MAD experiment using a lead (Pb) derivative. Nef interacts with the $\mathrm{SH} 3$ domain via a polyproline type II (PP-II) helix, which includes the conserved PxxP motif. This aspect of the interaction is almost precisely the same as that seen in the SH3:peptide interactions. In addition, there is a hydrophobic pocket on the surface of Nef that engages an isoleucine residue of the SH3 domain. It is this interaction that allows Nef to distinguish between closely related $\mathrm{SH} 2$ domains, and the binding pocket is formed by the anti-parallel arrangement of two a-helices that follow the PxxP motif and bracket it in the Nef tertiary structure.

The Src-family tyrosine kinases share a common regulatory mechanism that involves the SH2 and SH3 domains. The C-terminal region of each of the proteins consists of a short tail that follows the catalytic kinase domain, and the normal cellular form of the protein is phosphorylated on a tyrosine residue in this region. The down-regulated form of the Srcfamily tyrosine kinase Hck has recently been crystallized its structure determined, using MAD analysis of a mercury derivative (Sicheri et al., 1997). This crystal structure reveals 
that the SH3 domain plays the major role in distorting the catalytic domain to downregulate its activity, and that the role of the $\mathrm{SH} 2$ domain is primarily to position the SH3 domain properly. In collaboration with Todd Miller (SUNY, Stony Brook), it has been shown that Nef is a potent activator of the Hck-tyrosine kinase in vitro, and the same face of the SH3 domain that interacts with Nef makes critical inhibitory interactions in the downregulated form of the tyrosine kinase (Moaefi et al., 1996). Other work in signal transduction that has relied on synchrotron radiation and MAD analysis includes the structure of the Ser/Thr phosphatase PP1 (Goldberg et al., 1996) and the N-terminal domain of the STAT-1 molecule (Vinkemeier et al., in preparation). The structure of calmodulin-dependent protein kinase was determined using crystals that diffract only weakly. Utilization of synchrotron radiation at CHESS extended the resolution from $3.0 \AA$ (in house) to $2.5 \AA$ (CHESS) (Goldberg et al., 1996).

The laboratory is also involved in structural studies on the components of DNA polymerases that replicate chromosomes during cell division (supported by the National Institutes of Health, and carried out in collaboration with Mike O'Donnell, HHMI and Cornell Medical College, and Jerard Hurwitz, Sloan-Kettering Institute). These polymerases initiate replication in response to regulatory signals, achieve high processivity without dissociation from the template, and then disengage rapidly and restart replication elsewhere as needed. Proliferating cell nuclear antigen (PCNA) is the processivity factor required for chromosomal replication by eukaryotic DNA polymerase $d$. The crystal structure of yeast DNA polymerase d processivity factor PCNA (proliferating cell nuclear antigen), was determined using MAD analysis of a mercury derivative (Goldberg et al., 1996). The structure of human PCNA complexed with a 22 residue peptide derived from the C-terminus of the cell-cycle checkpoint protein p21 CIP1NAF1, has been determined at $2.6 \AA$ resolution, using data collected at beamline $X 25$, NSLS (Gulbis et al, 1996). The structure of the $c-y$ complex of $E$. coli DNA polymerase III was determined using MAD analysis of a mercury derivative, again at beamline $X 25$. The crystals utilized were very small, and absolutely required synchrotron radiation.

Very recent work has begun to focus on the components of the machinery responsible for nuclear transport. We have now obtained crystals of the b-type karyopherin molecule, which is thought to be responsible for the transport of ribosomal proteins. These crystals diffract to only $3.5 \AA$ on a rotating anode generator and have a unit cell axis in excess of $300 \AA$. The efficient solution of this structure will greatly benefit from the ability to utilize the intense, tunable radiation available from an insertion device.

Rationale for MAD Undulator. As is the case in Dr. Burley's research program, the use of tunable, high flux synchrotron radiation has proven invaluable to our own program. The research program described here will increasingly rely on bright MAD sources for a number of reasons. First, access to very bright radiation has enabled structure determinations to proceed in situations where the crystal quality was insufficient for conventional $X$-ray analysis. Second, and increasingly important, the utilization of cryogenic data collection has introduced a much greater level of non-isomorphism between crystals of a particular protein. While cryogenics is essential for preserving the diffraction of most of the crystals the laboratory has worked on, its utilization has made it much more difficult to obtain accurate phases using conventional MIR techniques. MAD analysis has therefore been utilized to get around this problem. In addition, sufficient heavy atom derivatives are not always obtainable, in these cases we have had success with Se-Met labeled protein 
(Martin et al., 1993). Lastly, the increasing interest in larger proteins and larger macromolecular complexes presumes weaker diffracting, small, and fewer crystals will be available. Thus, the increased source brightness provided by the small-gap undulator compared to focused bending magnet lines will be of significant benefit.

\section{References}

Goldberg, J., Nairn, A.C. \& Kuriyan, J. Structural basis for the autoinhibition of calcium/calmodulin-dependent protein kinase I. Cell 84, 875-887 (1996).

Gulbis, J., Kelman, Z., Hurwitz, J., O'Donnell, M. \& Kuriyan, J. Structure of the Cterminal region of $2^{1 \text { WAF1/CIP }} 1$ complexed with human PCNA. Cell 87, 297-306 (1996).

Krishna, T.S.R., Kong, X.-P., Gary, S., Burgers, P. \& Kuriyan, J. Crystal structure of the eukaryotic DNA polymerase processivity factor PCNA. Cell 79, 1233-1243 (1994).

Lee, C.-H., Saksela, K., Mirza, U.A., Chait, B.T. \& Kuriyan, J. Crystal structure of the conserved core of HIV-1 Nef complexed with a Src family SH3 domain. Cell $85,931-942$ (1996).

Martin, J.L., Bardwell, J.C.A. \& Kuriyan, J. Crystal structure of DsbA, a protein required for disulfide bond formation in Escherichia coli. Nature 365, 464-468 (1993).

Moarefi, I., LaFevre-Bernt, M., Sicheri, F., Huse, M., Lee, C.-H., Kuriyan, J. \& Miller, W.T. Maximal activation of a Src family tyrosine kinase Hck occurs by $\mathrm{SH} 3$ domain displacement. Nature, in press (1996).

Sicheri, F., Moarefi, I. \& Kuriyan, J. Crystal Structure of the Src-family tyrosine kinase Hck. Nature 385, 602-609 (1997). 
MAD Studies of Proteins that Control Cell Growth and Proliferation using a Small-Gap Undulator

\section{Dr. Nikola Pavletich, Sloan Kettering Institute}

Our laboratory is interested in the structural biology of pathways that control cell growth and proliferation, with particular emphasis on understanding signaling processes that regulate the eukaryotic cell cycle (Sherr \& Roberts, 1995). In these processes, signal integration and propagation relies heavily on protein-protein interactions, often involving the formation of macromolecular complexes of multiple components. Our aim is to use $X$ ray crystallography to understand (i) the specificity of macromolecular interactions in these systems, and (ii) how these interactions mediate signaling.

The eukaryotic cell cycle, which is the ordered progression of events such as growth, DNA replication, and cell division, fundamental to all eukaryotes (1), is coordinated by a family of related Ser/Thr protein kinases, the Cyclin-Dependent Kinases (CDKs). These kinases are regulated by multiple mechanisms: the CDK subunit requires the binding of its cyclin subunit for its activation; there are two distinct sites of phosphorylation, one activating, and two inhibitory ones, these modifications and their reversal being catalyzed by specific kinases (CAK and wee1) and phosphatases (cdc25); and there are CDK-inhibitory proteins, the Kip and INK4 families, that can bind CDKs and induce cell cycle arrest and differentiation (Morgan, 1995). Other regulatory processes include degradation, mediated by the eight-subunit cyclosome that binds and targets the mitotic cyclins to the proteosome in a cell cycle dependent manner (King et al., 1996); and conformational maturation (CDK4), mediated by the hsp90-cdc37 chaperone system (Stepanove et. al., 1996).

The substrates of the CDKs are not well understood yet, but several appear to be recruited to the kinase via stable protein-protein interactions at sites other than the catalytic cleft. For example, the p107 cell cycle regulator (Rb homologue) and the E2F1 cell cycle transcription factor can be isolated from cells in stable complexes with the CyclinA-CDK2 kinase (Zhu et al., 1995, Krek et al., 1994).

As part of our aim of understanding the structural biology of cell-cycle regulation, in collaboration with Joan Massague and Jerard Hurwitz (Sloan-Kettering), we have determined the crystal structures of the cyclinA-CDK2 (Jeffrey et al., 1995) and the phosphorylation-activated cyclinA-CDK2 (Russo et. al., 1996a) binary complexes, as well as the structure of the inhibited p27Kip1-cyclinA-CDK2 ternary complex (Russo et al., $1996 \mathrm{a}, \mathrm{b})$. As a prelude to understanding the maturation of CDK4 by hsp90, we have recently determined the structure of the putative polypeptide binding domain of hsp90 (Stebbins et. al., in preparation). To understand CDK regulation, our continuing studies are aimed at determining the structures of the p16INK4-CyclinD-CDK4, wee1-cyclinA-CDK2, cdc25-cyclinA-CDK2, cdc37-CDK4-hsp90 ternary complexes. We are also pursuing collaborative studies aimed at delineating those cyclosome components important for cyclin specificity and targeting as a prelude to crystallization. To understand CDK function, our research efforts are currently aimed at determining the structures of domains of the p107 and E2F1 substrates bound to the cyclinA-CDK2 complex. 
Rationale for MAD Undulator. The fundamental biological questions outlined above require the study of large multi-protein complexes, and for this we have to push the limits of both protein biochemistry and crystallography. In general our increasing interest in larger proteins and larger macromolecular complexes presumes that weaker diffracting, small, and fewer crystals will be available. Thus, the increased source brightness provided by the $X 13$ small-gap undulator compared to focused bending magnet lines will be of significant benefit. Today, the use of eukaryotic protein over-expression systems have allowed us to produce crystals of complex macromolecular assemblies, such as the ternary CyclinACDK2-p27Kip 1 complex (Russo et. al., 1996b). Importantly, recent advances have allowed for production of selenomethionyl substituted proteins in insect (Baculovirus) and mammalian (CHO) expression systems (Chen \& Bahl, 1991; Lustbader, et al., 1995). Crystals of these large assemblies often diffract poorly, and this presents a problem for both phase determination and precision in refinement. For example, our crystals of the CyclinA-CDK2 complex (Russo et al., 1996a), which had two complexes in an asymmetric unit and a large unit cell (space group P6222; $a=b=185, c=214$ ), diffracted at our home source to only 3.0-3.2 $\AA$. Phasing of this complex by the MIR method required over 30 data sets, and even then the phases were of marginal resolution, and refinement required data collection at a synchrotron source. The use of the MAD beamline would have significantly reduced both the time and the cost of this project.

\section{References}

Jeffrey, P. D. et al. Mechanism of CDK activation revealed by the structure of a cyclinA-CDK2 complex Nature 376, 313-320 (1995).

Krek, W. et al. Cell 78, 161-172 (1994) Negative regulation of the growthpromoting transcription factor E2F-1 by a stably bound cyclin A-dependent protein kinase.

Morgan, D. O., Nature 374, 131-134 (1995). Principles of CDK regulation.

Russo, A.A., P. D. Jeffrey, A. Patten, J. Massagué, N. P. Paveletich Nature 382, 325-331 (1996). Crystal structure of the p27Kip1 cyclin-dependent-kinase inhibitor bound to the cyclin A-Cdk2 complex.

Russo, A.A., P. D. Jeffrey, N. P. Pavletich Nature Structural Biology 3, 696-700 (1996). Structural basis of cyclin-dependent kinase activation by phosphorylation.

Stepanova, L., X. Leng, S. B. Parker, J. W. Harper Genes \& Dev. 10, 1491-1502 (1996). Mammalian p50Cdc37 is a protein kinase-targeting subunit of Hsp90 that binds and stabilizes Cdk4.

Zhu, L., E. Harlow, B. D. Dynlacht Genes \& Dev. 9, 1740-1752 (1995). p107 uses a p21CIP1-related domain to bind cyclin/cdk2 and regulate interactions with E2F. 
MAD Structural Analysis of Cell Surface Interactions

\section{Dr. Wayne A. Hendrickson, Columbia University and HHMI}

Biological problems. The major biological emphasis of our research is on protein interactions at the cell surface. Cells respond to their environment in large part through interactions involving proteins at their cell surfaces. These molecular events typically generate signals that traverse the cell membrane. While many proteins at the cell surface are integrally folded into the membrane, many others have single transmembrane segment with autonomously folded extramembranous domains. We produce recombinant fragments of these domains, expressed variously in bacteria, yeast, baculovirus-infected insect cells, or Chinese hamster ovary (CHO) cells (Hendrickson, 1996). Molecules currently under study include immune system receptors, growth factors and their receptors, protein kinases and associated signal transducers, and cell adhesion molecules.

Various extracellular components of the immune system are under study including T-cell receptors, class II MHC molecular, superantigens, the T-cell coreceptors CD4 and CD8, and appropriate complexes among them. In addition, we analyzing gp120 envelop protein of HIV in complexes with CD4 and antibody fragments. Continued efforts are also in progress on several growth factors or hormones and the ligand-binding domains of their receptors. These include stem cell factor, fibroblast growth factor in complex with heparin, and human chorionic gonadotropin, and ligand-receptor complexes including that with insulin. With respect to kinases, following on our initial structure of the insulin receptor tyrosine kinase, we are studying structures of the lymphocyte kinase which associates with CD4 and a trk kinase from nerve growth factor receptor. We are also attempting to produce and crystallize several protein kinase $C$ (PKC) isoforms and are studying the structural biology of PKC interacting protein (PKCI) and other HIT family proteins. Finally, the work on cell surface interactions, we are also engaged in a variety of other biological problems.

MAD methodology. In addition to our program of biological problems, we have been actively engaged in methology development and much of this work relates to the method of multiwavelength anomalous diffraction (MAD) for phase evaluation in crystallographic structure determination. We have devised effective experimental procedures for conducting MAD experiments and have developed a system of computer programs for analyzing the resulting data (Hendrickson, 1991). We also introduced the methodology for systematic incorporation of selenomethionine into proteins for MAD phasing (Hendrickson et al., 1990). Our effort in developing the HHMI Synchrotron Resource Facility at NSLS is an important component of our MAD research, and this has been instrumental in the recent dramatic increase in MAD phasing applications to problems of biological significance (Hendrickson and Ogata, 1997). Our first beamline, X4A which came into operation in late 1992, has produced approximately half of all MAD-phased structures with 32 novel structures published through 1996. As for MAD applications worldwide, a majority of these (19) were on selenomethionyl proteins.

Our development of MAD phasing technology has benefited greatly from applications to particular problems that are part of our biological research program. These studies have been carried at beamlines around the world, but in recent years the focus has been at the HHMI beamline $X 4 A$ at NSLS. During the last three years we have published structures 
based on MAD phasing for human chorionic gonadotropin (Wu et al., 1994), a tyrosine kinase fragment of the human insulin receptor (Hubbard et al., 1994), ciliary neurotrophic factor (McDonald et al., 1995), a catalytic fragment of MMLV reverse transcriptase (Georgiadis et al., 1995), an adhesive fragment of $\mathrm{N}$-cadherin (Shapiro et al., 1995), the biotinyl domain of acetyl-CoA carboxylase (Athappilly and Hendrickson, 1995), UmuD' (Peat et al., 1996), a substrate binding fragment of DnaK (Zhu et al., 1996), an adhesive fragment of myelin PO (Shapiro et al, 1996), a kinase fragment of Lck (Yamaguchi and Hendrickson, 1996), and FHIT (Lima et al., 1997).

Undulator impct. Among our recent MAD phasing experiences, there are two that involve undulator radiation. First, within months of the first usable beams at the European Synchrotron Radiation Facility (ESRF), we conducted a MAD-experiment on the Troika beamline. A fragment of $\mathrm{N}$-cadherin, which is a calcium-dependent cell adhesion molecule, was chosen for this study since crystals could be grown with ytterbium ions $\left(\mathrm{Yb}^{+3}\right.$ ) replacing $\mathrm{Ca}^{+2}$. The undulator gap was adjusted to place the third harmonic at the Yb LIIII edge $(8.9 \mathrm{keV})$, and we performed a four-wavelength experiment within the envelope at fixed undulator gap. We followed this with an experiment in which the ESRF accelerator group accommodated our calls for gap changes cycling between the edge energy and points at 50 and $700 \mathrm{eV}$ above the edge. This cadherin experiment was compromised by a poorly ordered $\mathrm{Yb}$ site and space group pseudosymmetry (P321 masquerading as P6322), but the polypeptide chain was nevertheless traced correctly (Shapiro et al., 1995). More recently, we determined the structure of FhIT by MAD phasing in one of the first experiments of any kind at the Advance Photon Source (APS). The structure analysis was made for the selenomethionyl protein with gap adjustments made on call to the control room (Lima et al., 1997).

The proposed small gap undulator will have an important impact on our program of biological research, and it will also be of great value to our program of MAD methology development. The intrinsic brightness of undulator radiation will make it possible for us to address ever increasingly challenging problems that may require strong focused beams to match small crystals, cope with large unit calls, and to make weakly diffracting crystals tractable. Moreover, the undulator brightness makes it feasible to use narrow bandpass monochromators with which we can investigate the impact on the signals from the white line features that occur from elements such as selenium, the lanthanides, tungsten and osmium.

\section{References:}

F.K. Athappilly and W.A. Hendrickson, "Structure of the Biotinyl Domain of AcetylCoA Carboxylase Determined Using MAD Phasing Techniques," Structure 3, 1407 1419 (1995).

M.M. Georgiadis, S.M. Jessen, C.M. Ogata, A. Telesnitsky, S. Goff and W.A. Hendrickson, "Mechanistic Implications from the Structure of a Catalytic Fragment of MMLV Reverse Transcriptase", Structure 3, 879-892 (1995). 
W.A. Hendrickson, "Determination of Macromolecular Structures from Anomalous Diffraction of Synchrotron Radiation", Science 254, 51-58 (1991).

W.A. Hendrickson, "Production of Crystallizable Fragments of Membrane Proteins", J. Bioenergetics and Biomembranes 28, 35-40 (1996).

W.A. Hendrickson and C.M. Ogata, "Phase Determination by the Method of multiwavelength Anomalous Diffraction (MAD)". Methods in Enzymology 276, 494523 (1997).

W.A. Hendrickson, J.R. Horton and D.M. LeMaster, "Selenomethionyl Proteins Produced for Analysis by Multiwavelength Anomalous Diffraction (MAD): A Vehicle for Direct Determination of Three-Dimensional Structure," EMBO J. 9,1665-1672 (1990).

S.R. Hubbard, L. Wei, L. Ellis and W.A. Hendrickson, "Crystal Structure of the Tyrosine Kinase Domain of the Human Insulin Receptor", Nature 372, 746-754 (1994).

C.D. Lima, K.L. D'Amico, I. Naday, G. Rosenbaum, E.M. Westbrook and W.A. Hendrickson, "MAD Analysis of FHIT, a Putative Human Tumor Suppressor from the HIT Protein Family", Structure 5, 763-774 (1997).

N.Q. McDonaid, N. Panayotatos and W.A. Hendrickson, "Crystal Structure of Dimeric Human Ciliary Neurotrophic Factor Determined by MAD Phasing", EMMBO J. 14, 2689-2699 (1995).

T.S. Peat, E.G. Frank, J.P. McDonald, A.S. Levine, R. Woodgate and W.A. Hendrickson, "Structure of the UmuD' Protein and its Regulation in Response to DNA Damage", Nature 380, 727-730 1996).

L. Shapiro, J.P. Doyle, P. Hensley, D.R. Colman and W.A. Hendrickson, "Crystal Structure of the Extracellular Domain from Po, the Major Structural Protein of Peripheral Nerve Myelin", Neuron 17, 435-449 (1996).

H. Wu, J. W. Lustbader, Y. Liu, R.E. Canfield and W.A. Hendrickson, "Structure of

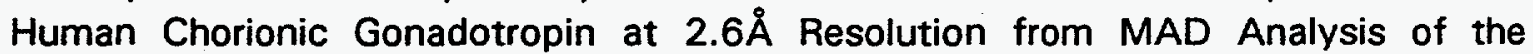
Selenomethionyl Protein", Structure 2, 545-558 (1994).

H. Yamaguchi and W.A. Hendrickson, "Structural Basis for Activation of Lymphocyte Kinase Lck upon Tyrosine Phosphorylation", Nature 384, 484-498 1996).

X. Zhu, X. Zhao, W.F. Burkholder, A. Gragerov, C.M. Ogata, M.E. Gottesman and W.A. Hendrickson, "Structural Analysis of Substrate Binding by the Molecular Chaperone DnaK, "Science 272, 1606-1614 (1996). 